\title{
Farmacoeconomia ed etica: cure sostenibili e rispetto della persona
}

\author{
Antonio G. Spagnolo*§, Roberta Minacori*
}

METODI

\begin{abstract}
The scarcity of economic resources today is a fundamental problem in public healthcare. The importance of making well-founded choices for optimal resources allocation concerns also pharmacological treatments and is justified also on ethical grounds, besides the economic, political and/or medical considerations. In fact, a better management, also in economic sense, of the patient and sickness involves the possibility of offering an efficient treatment to all the sick, or at least to as many of them as possible.

However, these considerations should always be subordinated to the ethical centrality of the patient, to the protection of his life, his health and his personal dignity, to the extent that life and health are priceless.

The goal of ethics in pharmacoeconomics can be summarised in respect for the person, which must remain the end and reference of every therapeutic choice as well as every healthcare policy.

The indispensable instruments for the realisation of the above objectives are, amongst others, those already highlighted but which demand further explanation: a) a suitable standard background/training and a multidisciplinary approach for optimising the evaluation procedures and increasing accuracy and verifiableness of the data; $b$ ) measures to contrast and disclose the conflict of interest for more transparency and credibility; c) a coherent evaluation of the ethical quality and humanness of the pharmacoeconomic analysis which requires a correct consideration of quality of life and a real respect for persons.
\end{abstract}

Farmeconomia e percorsi terapeutici 2004; 5 (4): 251-263

\section{INTRODUZIONE}

Scarsità o limitatezza delle risorse - che si tratti di personale, di tempo, di strutture, di finanziamenti - costituiscono, a fronte delle grandi prospettive di sviluppo della ricerca e della disponibilità di tecnologie medico-scientifiche sempre più all'avanguardia, la questione fondamentale, anzi il vero, grande, problema della sanità. Ciò comporta, e sarà così anche in futuro, per i "decision makers", a livello sia di macro-allocazione sia di microallocazione, il confronto con scelte operative relative alla distribuzione e alla migliore gestione possibile delle risorse disponibili. Fino a pochi anni fa, tali decisioni erano soprattutto il frutto di esperienze precedenti o di pareri raccolti da esperti, se non di puro istinto. La responsabilità di un'adeguata distribuzione delle limitate risorse di cui si dispone richiama, dunque, la necessità di analisi strutturate e sistematiche delle alternative e delle opzioni, prima di assumere decisioni operative in sanità.

L'importanza di operare tali scelte riguarda anche - e costituisce uno degli ambiti principali nella "razionalizzazione delle risorse in sa- nità" - i trattamenti farmacologici: gli esperti già da qualche tempo ammoniscono su tale esigenza di razionalizzazione, prevedendo per il futuro che non sarà possibile garantire a tutti ogni tipo di farmaco, soprattutto nel caso di farmaci che offrono benefici generici.

Tale necessità di una migliore distribuzione di risorse, quindi anche di un eventuale ripensamento nell'erogazione di determinati farmaci o nell' iter terapeutico, soprattutto per le patologie più frequenti e diffuse, trova giustificazione dal punto di vista sia medico, sia etico-deontologico e medico-legale, nel diritto/dovere di accertare l'efficacia e l'appropriatezza di una terapia prima di sostenerne, da parte del Servizio Sanitario Nazionale, l'accesso gratuito o a costi contenuti, e soprattutto nell'obiettivo di non sottrarre o diminuire opportunità diagnostico-terapeutiche per altri pazienti. Effettuare scelte di priorità d'impiego di farmaci, come stabilire delle priorità nella assegnazione di risorse sanitarie, potrebbe apparire in contrasto con l'imperativo etico-deontologico di tutelare la vita e la salute del singolo paziente; in realtà una migliore gestione, anche in senso economico, del malato
*Università Cattolica del Sacro Cuore, Facoltà di Medicina e chirurgia "A. Gemelli" Roma

§SIFEIT, Società Italiana per Studi di Economia ed Etica sul Farmaco e sugli Interventi Terapeutici 
e della malattia presume l'obiettivo di arrivare ad offrire la possibilità di un trattamento efficiente a tutti i malati, o almeno al maggior numero possibile.

Queste considerazioni, associate alle necessità di ordine economico delle politiche sanitarie, dovrebbero, però, sempre subordinarsi alla centralità etica del paziente, alle priorità correlate alla tutela della sua vita, della sua salute e della sua dignità personale.

\section{QUESTIONI EMERGENTI}

Finora, nell' ambito della valutazione complessiva dei farmaci, la rilevazione delle impressioni o delle preferenze del paziente-consumatore riguardo la gestione della propria salute non è stata tra le prerogative principali degli studi.

D'altra parte, l'esclusione o il non pieno coinvolgimento nelle valutazioni, cliniche ed economiche, di tutti i soggetti coinvolti e interessati dalle scelte allocative possono rendere, non infrequentemente, queste stesse scelte mal sopportate, e fonte di ulteriori fratture comunicative tra i cittadini-pazienti, le istituzioni e la stessa classe medica. Senza contare il fatto che effettivamente potrebbero essere attuati interventi sanitari economicamente più convenienti ma penalizzanti, almeno per certi aspetti, poco o nulla indagati ma ritenuti importanti dai pazienti.

Tali esigenze, non solo di ordine economico-politico ma anche medico ed etico, giustificano una rivisitazione del profilo di valutazione dei farmaci. Nuovi e già conosciuti prodotti sono oggetto di più approfondite revisioni: ai tradizionali criteri di efficacia e di sicurezza si affiancano parametri relativi alla convenienza economica e alla qualità della vita.

Difatti, gli studi di farmacoeconomia possono avere, proprio per l'imprinting di natura economica che li connota, lo svantaggio di fornire soprattutto dati quantitativi, con molta probabilità insufficienti, perciò, a delineare l'impatto qualitativo sulla singola persona (cioè tutti quegli aspetti importanti nella vita, personale, familiare, lavorativa, nella prospettiva dei pazienti).

Rispetto alle valutazioni operate in ambito farmacoeconomico, assume una rilevanza fondamentale la prospettiva, l'angolo di visuale utilizzata: ogni categoria interessata (il paziente, il medico, il farmacologo, l'economista, l'amministratore, il politico) ha un suo approccio e cerca di sottolineare alcuni aspetti. Ciò può comportare due conseguenze operative, una negativa, l'altra positiva, a seconda di quanto tutte le parti interessate riescano ad interagire e collaborare per cercare di raggiungere obiettivi comuni: l'affermazione o la predominanza di una prospettiva o di alcune e l'esclusione di altre, con la probabile deriva di tensioni, contrasti e scarsa compliance $\mathrm{o}$, seppure più difficile e impegnativo nell'attuazione, il reale coinvolgimento di tutte le parti, l' accoglienza di tutti gli apporti, la scelta di metodologie e obiettivi condivisi.

La farmacoeconomia, anche per la recente elaborazione come disciplina, si trova ancora in una fase di definizione dei suoi fondamenti teorici e di una metodologia o di metodologie che rispondano appieno ai criteri di rigorosità, precisione e riproducibilità scientifica, e attuabilità pratica, in un settore estremamente delicato e complesso qual è quello sanitario.

La dimostrazione della "utilità" e della convenienza di un trattamento farmacoterapeutico è diventata, dunque, un necessario completamento dell'efficacia clinica che, nella valutazione economica del farmaco, viene espressa generalmente dagli anni di vita guadagnati e dal miglioramento della qualità della vita. Gli studi di farmacoeconomia cercano, quindi, di rispondere ad alcuni specifici quesiti: quali farmaci devono essere inseriti in un prontuario terapeutico nazionale e quali in un prontuario terapeutico ospedaliero? Quale farmaco è più indicato, dal punto di vista medico ed economico, per un determinato paziente? Quale sostanza è la migliore candidata allo sviluppo per una industria farmaceutica?

A tali quesiti, se l'obiettivo è la ricerca del bene globale del paziente, dovrebbero, però, esserne aggiunti altri, inerenti la prospettiva dei pazienti: come l'uso di un farmaco piuttosto che un altro può influenzare la vita personale e familiare, o l'attività lavorativa? $\mathrm{Ci}$ sono dei casi in cui l'accettabilità "etica" di un farmaco, da parte del paziente o anche del medico "prescrittore", dovrebbe essere considerata insieme all' aspetto economico? Il medico "prescrittore" dovrebbe essere sollecitato, oltre che a contribuire efficacemente - come invitano a fare in questo periodo le Regioni - nel determinismo della spesa farmaceutica, anche a rilevare il grado di soddisfazione dei propri pazienti rispetto a modifiche terapeutiche "obbligate"?

E la farmacogenetica e la farmacogenomica, che attraverso lo studio della variabilità dei caratteri genetici si pongono l'obiettivo della personalizzazione della terapia farmacologica, o almeno di ottenere farmaci di cui si possa prevedere, per specifici gruppi genetici, il gra- 


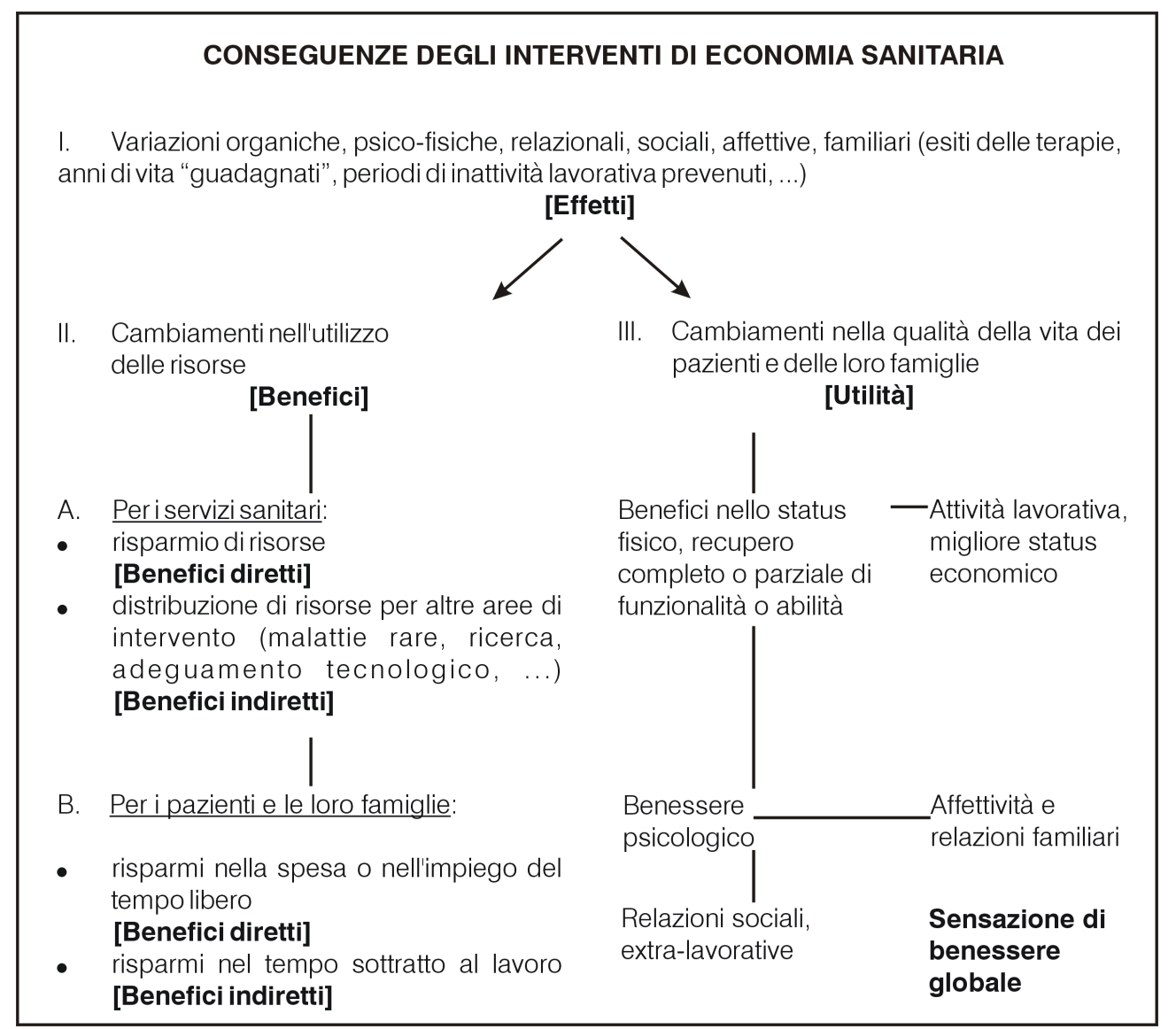

do di efficacia (o la non efficacia) o l'insorgenza di effetti indesiderati, che tipo di correlazione potranno avere con le valutazioni farmacoeconomiche nella scelta terapeutica? Almeno in ipotesi, sembrano prospettarsi finalità divergenti tra queste due aree: la farmacoeconomia tesa a integrare esigenze e a massificare costi e bisogni, la farmacogenetica a individualizzare la terapia, raggruppando i pazienti in base a determinati polimorfismi genetici. Anche se la farmacogenetica potrebbe consentire di risparmiare risorse, evitando l'utilizzo di farmaci di cui si può prevedere scarsità o assenza di efficacia terapeutica o insorgenza di pericolosi effetti collaterali.

Nell'ambito degli interventi, dei programmi e dei servizi sanitari l'identificazione e la misurazione dei costi, anche rispetto alle alternative, sono simili in tutte le analisi economiche. Più complessa è, invece, l'identificazione e la misurazione delle conseguenze, in termini di effetti, benefici e utilità, che possono risultare sostanzialmente differenti, considerata l'ampiezza e la varietà della materia [1] (fig. 1).

\section{LIVELLI DELLA DIMENSIONE ETICA IN FARMACOECONOMIA}

Anche per gli studi di farmacoeconomia, come per altri ambiti medico-scientifici, la va- lutazione etica investe sia l'ambito interno, metodologico, della disciplina stessa, sia quello esterno, con i risvolti e le possibili applicazioni dei dati ottenuti. Occorre considerare, cioè, che l'aspetto etico non può riguardare solo il momento applicativo, cioè il passaggio dai risultati degli studi alle decisioni allocative di priorità o di esclusione rispetto ai bisogni di salute delle persone, ma concerne anche quello della ricerca in farmacoeconomia. E non ci si riferisce solo alla cosiddetta etica intrinseca, relativa allo scrupolo metodologico, all'esattezza e veridicità dei dati, alla trasparenza dei procedimenti in modo che siano credibili e controllabili. Piuttosto, l'attenzione all' aspetto etico, dunque ai valori e ai principi in gioco, dovrebbe costituire un substrato senza soluzioni di continuità, anzi coerente, in tutte le fasi, nella progettualità, nella metodologia e negli obiettivi di uno studio di farmacoeconomia. Si è detto della necessità e della giustificabilità etica di approntare delle scelte in sanità, fondate su valutazioni scientificamente valide ed eticamente giustificate, per un'ottimizzazione dell'uso delle risorse e una loro equa e appropriata distribuzione, ma l'applicazione dei dati presuppone che essi siano validi scientificamente e rispecchino realmente tutti gli aspetti fondamentali necessari per esprimere decisioni che rispettino e tutelino tutte le persone coinvolte. Da quanto emerge dal dibat-
Figura 1

Tipologie di conseguenze da interventi di economia sanitaria (modificata da Drummond MF et al., 1993) 
tito presente nella letteratura internazionale, vi sono, nell'ambito degli studi di farmacoeconomia, alcuni aspetti critici, rilevanti per le conseguenze negative che potrebbero derivarne, e che meritano una riflessione etica.

\section{LA QUESTIONE DELL'APPROCCIO} MULTIDISCIPLINARE IN FARMACOECONOMIA E LA NECESSITÀ DI UN ADEGUATO TRAINING

Data la crescente richiesta di studi di farmacoeconomia, da più parti è stata sollecitata una particolare attenzione all' aspetto formativo riguardante questa disciplina. Recenti survey hanno evidenziato il problema della mancanza di adeguate conoscenze in questo ambito [2], addirittura di inadeguata competenza anche tra professionisti del settore farmaceutico e farmacologico [3], persino in paesi dove la farmacoeconomia si è affermata da più tempo come strumento di politica sanitaria [4]. In Giappone alcuni Autori hanno individuato nella limitata conoscenza e nella rarità di esperti l'ostacolo maggiore all'utilizzo delle valutazioni economiche in sanità [5].

Anche il tema della globalizzazione delle questioni relative ai servizi sanitari ha una crescente rilevanza a questo proposito. Molte industrie farmaceutiche di diverse nazioni si stanno fondendo, si moltiplicano le conferenze e le riviste specializzate che, nella partecipazione e nelle finalità, affermano interessi di respiro internazionale, sorgono anche gruppi internazionali per la difesa dei diritti di pazienti [6]. Sebbene una standardizzazione internazionale del training educativo-formativo e della ricerca in farmacoeconomia possa essere un obiettivo utile e raggiungibile, le differenze e specificità delle singole nazioni non dovrebbero essere ignorate, anzi a volte dovrebbero essere attentamente considerate e tutelate. Dunque, nonostante l'opportunità di elaborare obiettivi formativi comuni, teorie e metodologie dovrebbero essere utilizzate come una base, un core fondamentale di conoscenze, a cui associare elementi applicativi specifici e fattori integrativi, variabili secondo le differenze locali, relativi a costi, risorse, particolarità demografiche e culturali, condizioni di sviluppo economico, modelli di assistenza, sistemi sanitari, assicurazioni e aspettative dei pazienti.

Comunque, a differenza di altri ambiti scientifici, non esistono attualmente standard background/training in farmacoeconomia; ciò perché l'area della farmacoeconomia comprende alcuni ambiti dell'economia sanitaria ma utilizza valutazioni di carattere clinico, farmacologico e umanistico [7,8] (fig. 2).

La farmacoeconomia, infatti, può anche comprendere ricerche non economiche, come le valutazioni di risultati di studi clinici o psicosociali o di altri ambiti umanistici, quali quelli sulla qualità della vita [9]. I ricercatori nell'ambito della farmacoeconomia possono, inoltre, provenire da diverse aree formative. È stata anche evidenziata, a questo proposito, la possibilità di attribuire più qualifiche a chi studia la farmacoeconomia (farmacoeconomista, economista sanitario, socioeconomista, ricercatore di risultati) [10]. Dunque, poiché la ricerca in farmacoeconomia comprende competenze derivanti da diverse discipline, sarebbe essenziale un team di ricerca multidisciplinare. Tra le discipline con un ruolo-chiave vi sarebbero l'economia, la medicina, la farmacia, l'etica, l'epidemiologia, l'organizzazione dei servizi sanitari, la sociologia, la psicometria, la statistica [11, 12].

Nel 1998 un panel di membri dell'International Society for Pharmacoeconomics and Outcomes Research (ISPOR), convenuti per discutere sul tipo di formazione e sulle competenze necessarie per condurre, interpretare e utilizzare le valutazioni economiche in sanità, aveva individuato tre livelli di competenza in farmacoeconomia:

"consapevolezza", vale a dire piena padronanza della disciplina, in senso teorico e pratico; obiettivo raggiungibile attraverso un training continuo o con corsi di breve durata;
Figura 2

Rappresentazione schematica della sovrapposizione tra farmacoeconomia, economia sanitaria e risultati della clinica, della farmacologia e di altre scienze umane (psicologia, sociologia, bioetica,...) (da:Rascati $\mathrm{KL}$, Drummond MF, Annemans $L$ and Davey PG, 2004)

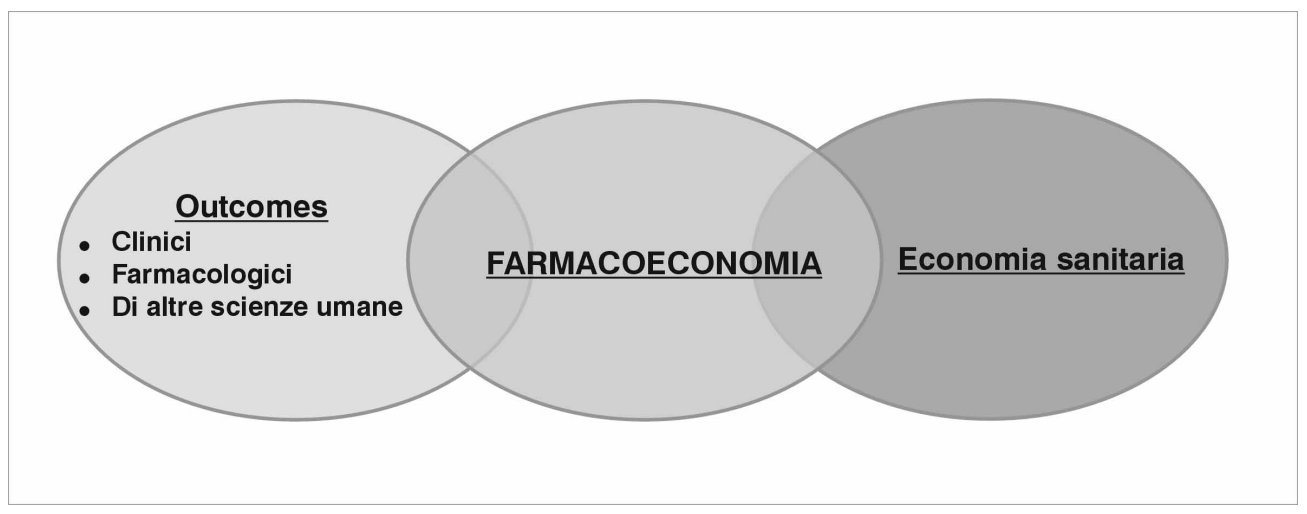




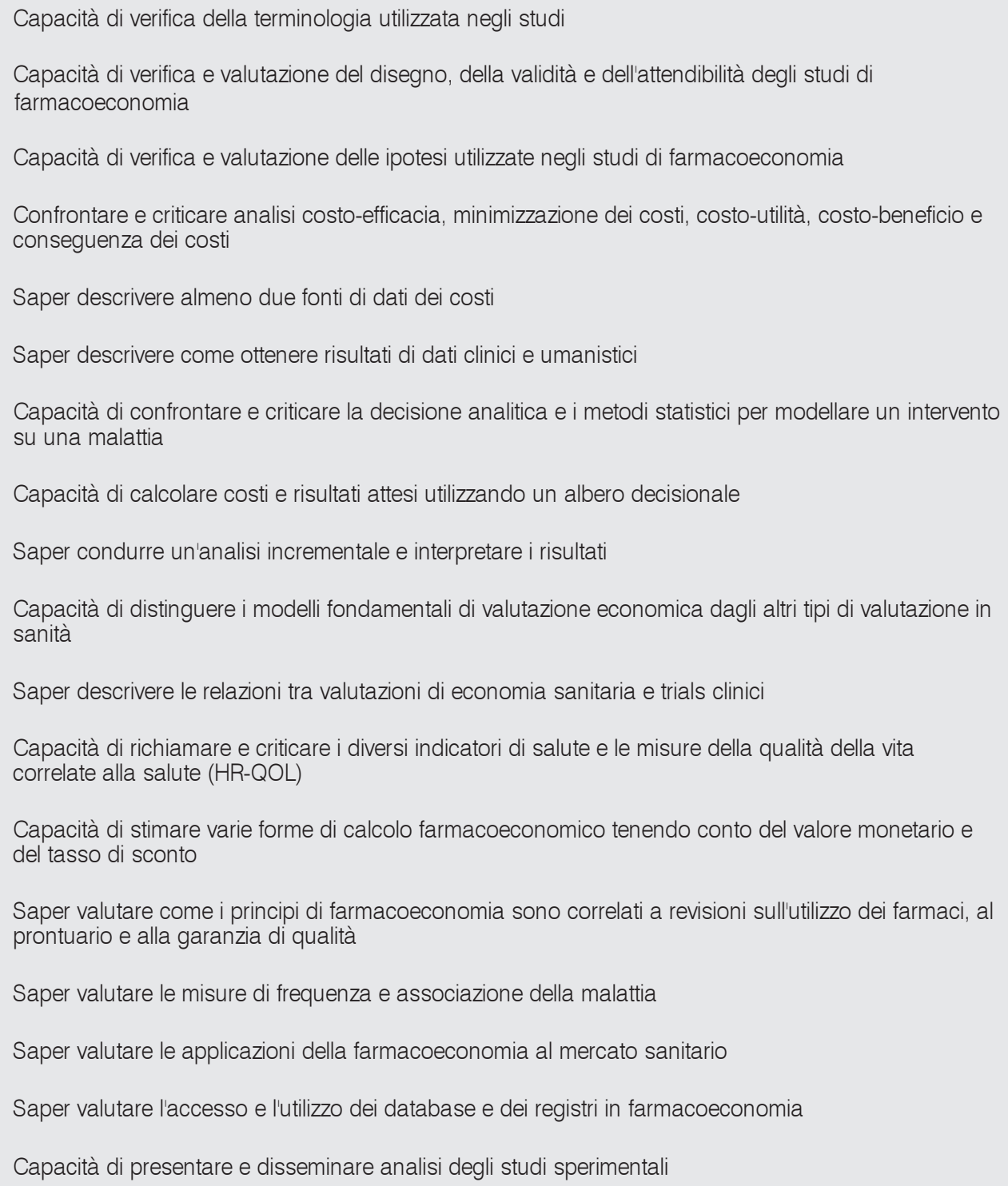

"applicazione", intesa come la capacità di confrontare in modo critico, valutare $\mathrm{e}$ prendere decisioni basate sulla ricerca economica; raggiungibile tramite esperienza diretta e formazione di livello superiore;

"concettualizzazione", vale a dire capacità di realizzare nuove metodologie di analisi e di sviluppare teorie, assimilando da altre discipline; tale obiettivo sarebbe ottenibile attraverso specifici corsi post-lauream [13].

È stata anche evidenziata una certa preoccupazione sulla capacità di istituzioni accademiche e industrie farmaceutiche di soddisfare la crescente richiesta di qualificati professionisti in farmacoeconomia. Paltiel e Neumann hanno raccomandato che, finché non si raggiunga un numero soddisfacente di specialisti di farmacoeconomia laureati, dovrebbe essere richiesto un avanzato curriculum da integrare con formazione e aggiornamento continui [14].
Una task force formata nell'ambito dell'ISPOR nel 2001, seguendo un documento definito nel 1996, ha tracciato una lista di obiettivi e di risultati per il training in farmacoeconomia arrivando ad approvare nel 2002 una lista di 18 obiettivi formativi [15], elencati in tabella 1 .

Nonostante tale definizione di obiettivi, di fondamentale importanza, non esiste ancora uno standard formativo condiviso che un "farmacoeconomista" dovrebbe possedere: è ovvia la difficoltà di essere esperti in tutte le discipline coinvolte in questo campo. Perciò, si raccomanda che nell'ambito di progetti di ricerca in farmacoeconomia vi sia un team multidisciplinare, in cui coesistano, nei vari membri, diversi livelli di competenza (tra consapevolezza, applicazione e concettualizzazione). Una previsione di reale efficacia necessita, comunque, che ogni elemento del team abbia sufficiente conoscenza delle altre discipline affinché sia possibile una reale interazione tra le diverse competenze.
Tabella 1

Obiettivi formativi in farmacoeconomia proposti dalla task force dell'International Society for outcomes research (ISPOR) (Marsh, 2002) 
LA VALUTAZIONE DELLA QUALITÀ DELLA VITA, L'INFORMAZIONE E IL CONSENSO NEGLI STUDI DI FARMACOECONOMIA: LA PROSPETTIVAETICA

Il primo quesito da porsi, riflettendo su tale ambito della ricerca biomedica, riguarda la giustificazione della conduzione di questa ulteriore valutazione.

Nella comunità scientifica e nella società vi è generale consenso sull'esigenza di una ricerca biomedica che sia sempre giustificata sufficientemente sul piano scientifico ed etico. Negli Stati Uniti, dopo la denuncia di vari, gravissimi, abusi perpetrati su persone partecipanti a sperimentazioni cliniche, la National Commission for the Protection of Human Subjects of Biomedical and Behavioral Research affermava tale consenso con queste parole: "I soggetti non dovrebbero essere esposti a rischi in sperimentazioni disegnate in modo così inadeguato che gli obiettivi prefissati non possano essere comunque ottenuti (nostra la traduzione)" [16].

Lo scopo della ricerca clinica è l'innovazione terapeutica - che è un bene per la società e per le singole persone -, vale a dire ottenere nuove terapie in grado di guarire, curare o prevenire le malattie o migliorarne il loro controllo. Tale finalità medico-scientifica è la traduzione di un principio etico fondamentale, il principio della beneficità (beneficence) che ha contraddistinto da sempre la medicina e la figura stessa del medico. Negli USA la già citata National Commission, in un famosissimo documento, spiegava tale principio in due regole generali: "non arrecare danno, massimizzare i possibili benefici e minimizzare i possibili danni" [17]. Nel contesto della medicina tale principio è stato espresso dalla massima "primum non nocere" e da un'espressione presente nel Giuramento di Ippocrate ("porrò in essere trattamenti per aiutare il malato secondo la mia capacità e il mio giudizio“). Nella ricerca biomedica, prima del Belmont Report, è stata la Dichiarazione di Helsinki, emanata dall' Assemblea della World Medical Association a raccomandare che il medico-ricercatore non solo sia garante del benessere degli individui (soggetti di ricerca e pazienti) ma anche si adoperi affinché tale compito sia svolto in modo sempre migliore. In precedenza, nel Codice di Norimberga si era affermato che i rischi derivanti dalla sperimentazione devono essere giustificati “dall'importanza umanitaria del problema che dovrebbe essere risolto dalla sperimentazione" [18].
La ricerca nell'ambito dell'innovazione terapeutica si focalizza su "misure di risultati o endpoints che sono considerati obiettivi per quanto essi siano basati su osservazioni esterne del paziente" [19, p. 115]. In oncologia, ad esempio, misure importanti del risultato sono costituiti dalla riduzione della massa tumorale e dalla persistenza delle remissioni, mentre nella sperimentazione sugli anti-ipertensivi il dato più importante è la riduzione della pressione diastolica. Altri dati quantitativi importanti sono quelli relativi agli eventi avversi sofferti dai pazienti-soggetti di sperimentazione.

Tali dati sono fondamentali, ma non esauriscono tutta l'importanza della medicina, soprattutto nella prospettiva dei pazienti. Essi hanno un punto di vista del proprio benessere, presente e futuro, più ampio, con aspettative che vanno al di là dei dati, così importanti per i medici. I pazienti si possono porre domande ben diverse dai quesiti dei medici: "Come cambierà la mia vita se prenderò questo farmaco? Cosa sarò in grado di fare durante l'assunzione? Come mi sentirò? E se non lo prendessi?". Si tratta di dubbi, quesiti, dunque, che riguardano non dati quantitativi ma la "qualità della vita" dei soggetti. Tali quesiti conducono ad un altro principio basilare nell'etica della ricerca clinica: il rispetto per le persone. Tale principio, secondo l'interpretazione data dalla statunitense National Commission, si materializza nel riconoscimento e nel rispetto dell' autonomia delle persone e nella protezione dei soggetti incapaci [18].

Il consenso informato rappresenta lo strumento, per l'ambito etico-deontologico e del diritto, attraverso cui il medico-ricercatore evidenzia tale rispetto per le persone, per la loro autonomia. Tale strumento, prima della deliberazione del paziente-soggetto, prevede la presentazione delle informazioni relative, tra le altre cose, ad eventuali rischi, benefici e alternative. Ciò che è importante perché vi sia reale riconoscimento e rispetto per l'autonomia, o, come enunciato nella bioetica personalista, la libertà-responsabilità delle persone, è che non solo tale informazione comprenda tutti gli aspetti rilevanti della sperimentazione e che sia comunicata in modo idoneo e perciò compresa, ma che trasmetta significati, fornisca chiarimenti che sono rilevanti rispetto alle preoccupazioni dei pazienti. L'informazione, in pratica, dovrebbe cercare di corrispondere a quelle domande, prima citate, forse poco significative per il medico, ma molto importanti per le persone. Le persone, infatti, normalmente hanno dei progetti per la propria vita, il proprio lavoro, la propria famiglia: si tratta della personale, unica, prospettiva di 
ciò che è importante portare a termine o incominciare a fare, del come condurre una vita soddisfacente. La contingenza negativa della malattia viene inesorabilmente ad alterare, in varia misura, tale prospettiva, richiamando ad una riconsiderazione e rivalutazione dei propri progetti. I pazienti, dunque, hanno bisogno di rapportare queste esigenze, questi significati, alla malattia, alle terapie che verranno proposte, al percorso che saranno invitati a fare. Un reale rispetto per le persone chiama, dunque, il medico e tutti coloro che sono coinvolti nella finalità di ottenere la miglior cura a rispondere anche a questi quesiti, a sforzarsi di raggiungere, cioè, l'obiettivo più ampio, e anche più complesso, di curare tutta la persona, nella sua globalità, e a non soffermarsi solo sulla malattia.

In genere, nel corso dello sviluppo di una nuova terapia è possibile prevedere quando la sua somministrazione comporterà un condizionamento o un'influenza che potrebbe essere qualitativamente rilevante sulle attività quotidiane o sulla vita delle persone. Una sperimentazione che trascurasse a priori di considerare tale aspetto, quando la rilevanza di tale questione può essere prevedibile, dovrebbe essere considerata inadeguata rispetto alla finalità propria della ricerca di ottenere una migliore cura. E dal punto di vista etico se non anche scientifico - da ritenere poco o per nulla giustificabile. Infatti, verrebbero ad essere messi in discussione due capisaldi etici rilevanti: l'obiettivo del raggiungimento del maggior benessere dei pazienti e la possibilità di mettere a loro disposizione informazioni utili a rendere più consapevole e libera la scelta di assumere determinati trattamenti.

Schipper e Clinch hanno così espresso le loro simili riflessioni in proposito: "in definitiva, un trial clinico randomizzato disegnato in modo accurato, ben condotto, può fornire una evidenza statistica di una differenza di risultato. Ma tale evidenza è matematica. Rimane il dovere per il ricercatore di assicurare che la 'significatività' abbia importanza biologica e umana" [20, p. 116].

\section{QUALE RIFERIMENTO ETICO PER LA "QUALITÀ DELLA VITA"?}

Quando si fa riferimento all'espressione "qualità della vita" occorre, innanzitutto, evidenziare che tale locuzione sottende una varietà di significati ed interpretazioni.

Le prospettive etiche interpretative sono sostanzialmente quattro. La prospettiva libertaria individualistica, il cui valore di riferimento è la libertà dell'individuo, colto nella sua inviolabile autodeterminazione, e che è riconducibile alla teoria della "soddisfazione delle preferenze (preference satisfaction)": in questa prospettiva una vita piacevole (good life) consiste nella soddisfazione di desideri e preferenze delle persone.

La prospettiva dell'utilitarismo che tenta di ricercare l'uguale considerazione degli interessi soggettivi, le cosiddette utilità, e secondo la quale occorre massimizzare la felicità e minimizzare il dolore (the edonist) per le persone che possono essere consapevoli di determinate esperienze [20]. Si tratta della ricerca del miglior saldo attivo della bilancia benessere/dolore e della qualità della vita attraverso analisi costo-utilità, la cui stima è espressa generalmente in anni di vita guadagnati o in anni caratterizzati da determinati indici di qualità rispetto ad un ventaglio di aree funzionali (fisica, psichica, interazione sociale, condizione economica, ...) o di attività quotidiane (alimentazione, relazionalità, lavoro, ...) o di fattori ritenuti indispensabili per la realizzazione di aspirazioni personali e del benessere psicofisico e socio-relazionale.

Un'altra interpretazione teorica sostiene che, almeno in parte, una vita piacevole non consiste né in esperienze consapevoli di edonismo né nella soddisfazione di preferenze o desideri, ma nella realizzazione di specifici ideali normativi. Ad esempio, alcuni hanno sostenuto che una componente di una vita piacevole consiste nell'essere un agente autonomo e che si auto-determina, e che ciò può tradursi in una vita piacevole anche se il soggetto non è più felice grazie a questo e non ha il desiderio di essere autonomo [21].

La prospettiva etica a cui vogliamo, invece, riferirci pone al centro la persona umana nella sua uni-totalità di corpo e intelletto, di soggettività e di coscienza morale, di unicità e di irripetibilità, di relazione e di interpretazione della realtà, radicalmente nel suo esistere, nel suo esserci [21,22]. Ciò detto per chiarire che l'aspetto della qualità della vita rappresenta un elemento rilevante dal punto di vista etico, ma che non può e non deve esaurire tutto il valore della persona umana, della sua vita e della sua dignità.

Dunque, se è importante, in certi contesti anche determinante, compiere delle valutazioni che concernono le conseguenze di orientamenti clinici o di analisi farmacoeconomiche sulla qualità della vita degli interessati, è imprescindibile però che la valutazione sulla qualità della vita è da ritenere secondaria al valore della vita dell'individuo. Attraverso tali valutazioni, infatti, possiamo operare un giudizio 
di qualificazione delle funzionalità, della capacità, ma la qualità non può sostituire o essere sovrapponibile all'essenza valoriale, all'ontologia dell'individuo umano. Le misure della qualità della vita nel contesto sanitario e nello specifico della farmacoeconomia rappresentano utili strumenti che possono contribuire in modo determinante a raggiungere l'obiettivo del miglioramento della cura e di una maggiore corrispondenza alle aspettative dei pazienti, ma nessun algoritmo che registri funzionalità o produttività, desideri e preferenze dei soggetti o utilità sociali può indurre a legittimare arbitrarie discriminazioni tra malati.

Inoltre, è anche da considerare che le valutazioni sulla qualità della vita derivanti da espressioni di preferenza o di aspettative dei pazienti, della loro libertà-responsabilità, della loro capacità e volontà di auto-determinarsi, lascia esclusi tutti quei soggetti "deboli" in quanto incapaci di esprimere volontà, libertà o preferenze, quali i bambini, gli adulti incapaci per patologie mentali, le persone in stato vegetativo o comatose: chi potrebbe legittimamente, in loro vece, esprimere preferenze sulla loro qualità della vita?

\section{CRITERI PER DETERMINARE LA NE- CESSITÀ DI VALUTAZIONI SULLA QUALITÀ DELLA VITA}

Le valutazioni sulla qualità della vita vengono richieste per alcune ma non per tutte le sperimentazioni di nuovi farmaci. In generale, tali valutazioni sarebbero necessarie quando si può ragionevolmente prevedere che $\mathrm{i}$ risultati forniranno informazioni che saranno di "utilità pratica" a medici e pazienti nella scelta di utilizzare il nuovo farmaco e quando l'importanza di tale dato giustifica i costi e i rischi della sperimentazione. Levine indica l'opportunità di compiere tali valutazioni come un dovere prima facie, cioè un' azione obbligatoria a meno che vi sia un conflitto con altri doveri più importanti o a meno che vi sia una giustificazione etica a soprassedere a tale dovere [19]. L'esigenza di condurre valutazioni sulla qualità della vita verrebbe meno se le condizioni previe e quanto è già conosciuto non giustificano né i costi da sostenere né i rischi per i soggetti.

La previsione dell'insorgenza di effetti collaterali verosimilmente in grado di influire negativamente sulla qualità della vita dei soggetti è una delle condizioni che esige necessariamente delle valutazioni sulla qualità della vita nell' ambito della sperimentazione clinica, tanto più se si tratta di patologie croniche o di effetti a lungo termine [23].
Il criterio dell' "utilità pratica" è strettamente correlato al concetto di "rischio materiale", utilizzato nel diritto nell' ambito della dottrina sul consenso informato. Negli Stati Uniti, in una sentenza storica per quanto concerne il consenso informato (Canterbury vs Spence [24], la corte aveva affermato che un rischio è "materiale" se l'informazione che lo riguarda ha un'utilità pratica nell'elaborazione della decisione di assumere o rifiutare una terapia proposta. Anche dal punto di vista etico, tale concetto necessita di una riflessione. Alcuni esempi di situazioni in cui l'informazione relativa alla qualità della vita potrebbe risultare di grande utilità possono aiutare a esplicitare l'esigenza di tale specifica valutazione:

il caso della terapia anti-ipertensiva nell'ipertensione lieve, quando i pazienti devono ben recepire l'importanza di ottenere una piccola riduzione nella probabilità di insorgenza di eventi morbosi seri [25];

la somministrazione di chemioterapia a forte tossicità che può offrire una piccola chance di remissione a pazienti con neoplasie altamente refrattarie ma che può diminuire la qualità (o anche la quantità) di vita;

un ultimo esempio è quello della possibilità di attuare due opzioni terapeutiche che possono condurre allo stesso risultato in termini di sopravvivenza, ma che comportano sostanziali differenze nella qualità di vita (ad es. intervento chirurgico radicale vs. intervento chirurgico conservativo associato a chemioterapia) [26].

Secondo altri autori le valutazioni sulla qualità della vita sarebbero generalmente non necessarie nel caso di somministrazione farmaci salva-vita o in grado di ripristinare le condizioni di vita normale come prima della malattia [27]. Molto interessante, al riguardo, uno studio di McNeil e coll. su 49 volontari sani (37 manager e 12 vigili del fuoco con un età media di 40 anni), cui era stato chiesto di immaginarsi affetti da cancro al laringe e di dover prendere delle decisioni sulla terapia di tipo conservativo (per preservare la voce) o più radicale (per aumentare le probabilità di sopravvivenza). Ebbene, nonostante la maggior parte di essi avrebbe accettato una lieve riduzione nella sopravvivenza a lungo termine pur di mantenere la propria voce, nessuno avrebbe virtualmente accettato una diminuzione della sopravvivenza sotto i 5 anni. Sulla base di altri dati concordanti con questo, gli autori concludevano che le scelte sui trattamenti da parte dei pazienti erano basati non solo sulla qualità ma anche sulla quantità di vita prospettata [28]. 
Un criterio supplementare per determinare la necessità di studi sulla qualità della vita è quello economico, teso a individuare trattamenti troppo onerosi per la società e al contempo per i pazienti stessi, per quanto concerne la qualità della vita, e a selezionare terapie che assicurino la maggiore e migliore aspettativa di vita possibile rispetto alla risorse investite [29]. La finalità di tale criterio è giustificabile nell'ottica di una migliore allocazione delle risorse, soprattutto nel contesto di una spesa sanitaria crescente e di bisogni sanitari non sempre coperti. Il criterio economico, però, per preservare tale giustificazione applicativa - in una sanità che considera realmente come sua finalità principale $\mathrm{e}$ superiore la cura dell'individuo umano, soprattutto quello più debole, malato e bisognoso di protezione - non dovrà condizionare tout court una scelta terapeutica, nel caso questa fosse ritenuta efficace dal medico e accettata dal paziente.

\section{CONFLITTO DI INTERESSI, TRASPA- RENZA E CREDIBILITÀ DEGLISTUDI DI FARMACOECONOMIA}

La validità e credibilità delle valutazioni di farmacoeconomia è attualmente argomento di grande dibattito. Lo scenario che fa da sfondo a tale situazione è alquanto complesso: da una parte la necessità di contenere i costi per la spesa sanitaria, per i continui tagli alle risorse disponibili, dall'altra bisogni sanitari che aumentano e costano sempre più; a ciò si aggiungano anche gli interessi commerciali (legittimi) delle industrie farmaceutiche che cercano di non perdere quota in un "mercato" sempre più difficile. È, perciò, ovvio che queste ultime si adoperino per evidenziare i benefici clinici ed economici dei propri prodotti, anche attraverso specifiche valutazioni quali quelle farmacoeconomiche per fare pressione, come successo nel Regno Unito, in Canada e in Australia [30,31,32 ], sulle istituzioni responsabili dell' elaborazione dei prontuari farmaceutici. Da ciò si può comprendere il peso che potrebbe essere attribuito ai risultati degli studi di farmacoeconomia e quanto sia importante dirimere la questione del conflitto di interesse, che pure è un problema chiave della ricerca biomedica.

Qualche anno fa, Friedberg et al., attraverso una revisione di articoli pubblicati su analisi di costi e costo-efficacia di 3 classi di farmaci oncologici (fattori di stimolazione ematopoietica, antiemetici antagonisti della serotonina e taxani), hanno evidenziato come gli studi condotti con finanziamento delle in- dustrie farmaceutiche abbiano ottenuto dati qualitativi conclusivi non favorevoli con una proporzione quasi 8 volte minore $(1 / 20-5 \%$ vs. $9 / 24-38 \%$ ) rispetto a studi analoghi non sponsorizzati, mentre i risultati favorevoli, sempre da studi finanziati dalle industrie, sono stati di 1,4 volte maggiore rispetto agli studi indipendenti. Gli Autori hanno formulato una serie di ipotesi per spiegare i risultati della loro indagine: la possibilità per gli sponsor di individuare e selezionare, dopo i primi risultati clinici, le sperimentazioni che hanno maggiori possibilità di ottenere dati favorevoli e di decidere di sostenere, anche attraverso gli studi di farmacoeconomia, questi trials piuttosto che altri verosimilmente destinati a fornire risultati sfavorevoli; la presenza di evidenti bias nella ricerca farmacoeconomica che mostrava di preferire la pubblicazione di studi con dati "positivi"; le influenze multiformi delle industrie farmaceutiche sulla sperimentazione, attraverso l'erogazione di fondi, aiuti, benefits di vario tipo o di accordi con i ricercatori per la pubblicazione dei risultati; la possibilità per le industrie farmaceutiche di collaborare direttamente con i ricercatori nell'elaborazione dei protocolli di analisi economiche e di influenzare indirettamente la scelta dei criteri di valutazione economica [33]. Tale strana contingenza favorevole tra sponsorizzazione e risultati positivi ha, d'altronde, sollevato dubbi e incertezze nell'opinione pubblica [34,35].

In uno studio pubblicato nel 2000, Hill et al., dell'Università di Newcastle nel New South Galles, Australia e del Pharmaceutical Evaluation Section of the Australian Pharmaceutical Benefits Scheme, aggiungevano a questo già fosco quadro altri contributi attraverso la revisione di 326 studi effettuati tra il 1994 e il 1997. In quella nazione vengono inseriti nel prontuario e pagati dalla sanità pubblica solo nuovi farmaci le cui analisi farmacoeconomiche indicano che sono costoefficaci per alcuni pazienti. Tra queste analisi, in ben il 67\% (218) degli studi erano stati individuati "seri problemi di interpretazione". In alcuni casi non erano disponibili dati da sperimentazioni controllate e randomizzate o queste erano di scarso rilievo statistico o qualitativo, o i calcoli dei costi e dei risultati non erano "trasparenti" [36].

Altri Autori in precedenza avevano già rilevato diversi bias nelle analisi costo-efficacia dei farmaci: Udvarhelyi et al. nel 1992 avevano evidenziato che le regole per tali valutazioni, da tempo conosciute nella letteratura medica, erano ampiamente contravvenute e i principi fondamentali ignorati [37]; Hillman et al., attraverso la loro esperienza di conduzione di 33 
analisi economiche per 15 industrie farmaceutiche per 13 anni, erano stati i primi a presentare i problemi dei bias nelle analisi costo-efficacia finanziati dai produttori di farmaci. Essi notarono che le industrie farmaceutiche sponsorizzavano la maggior parte di tali studi, che di frequente erano senza regolamenti né standard e inclini a bias soggettivi. In quegli studi, inoltre, i ricercatori, ricevevano dallo sponsor il protocollo praticamente già definito: erano già scelti il disegno e la metodologia, il farmaco di confronto, i dati confidenziali visionabili. Le industrie, rilevavano ancora gli Autori, disponevano di apposite marketing divisions che non si ponevano alcun problema a selezionare come confronto un farmaco non efficace, o a fare concludere precocemente e non pubblicare studi non favorevoli, o a mettere pressione sullo sperimentatore per produrre risultati favorevoli [38].

Inoltre, nonostante le sperimentazioni controllate e randomizzate siano considerate il "gold standard" per la ricerca clinica, esse hanno anche il limite di misurare l'efficacia in una parte deliberatamente ristretta della popolazione, e di non rispecchiare, quindi, adeguatamente l'effettiva applicabilità nella pratica clinica, che è l'area di interesse della farmacoeconomia [39]. Dunque, vi potrebbe essere un problema di inadeguatezza progettuale e metodologica, celata da una conduzione corretta, anch'essa derivante da motivi e influenze commerciali.

Comunque, anche se sono necessarie altre sorgenti finanziarie per gli studi di farmacoeconomia, limitare la pubblicazione degli studi sponsorizzati non è, probabilmente, né realizzabile né utile, dato che l'industria farmaceutica fornisce fondamentali risorse a molte aree della ricerca medica ed è oggi la principale risorsa per gli studi di farmacoeconomia.

Per migliorare la credibilità delle analisi economiche, dovrebbero essere perseguite politiche che promuovano una completa rivelazione di tutti gli eventuali interessi finanziari.

Anche la scelta di condurre un maggior numero di analisi prospettiche di farmacoeconomia (congiuntamente a sperimentazioni di fase III) potrebbe aumentare la credibilità degli studi stessi e dei dati ottenuti, poiché si eliminerebbe l'opportunità dei finanziamenti "selettivi" basati sulla "convenienza" dei risultati. Soprattutto tali analisi dovrebbero essere previste sin dalla prima elaborazione del disegno del trial, piuttosto che aggiungere successivamente delle variabili economiche alla struttura iniziale del protocollo (il cosiddetto "piggyback arrangement") [40].

Infatti, le valutazioni di ordine clinico ed economico comportano sostanziali differenze nel disegno, nella significatività statistica del campione (per le valutazioni economiche sarebbero, infatti, necessari campioni molto più ampi di popolazione) e nei costi da sostenere. Bower et al., riportando i risultati del loro studio, controllato e randomizzato, sulle psicoterapie nella depressione, che comprendevano nel disegno un' analisi costo-efficacia, hanno affermato che il campione di popolazione incluso per ottenere il principale obiettivo clinico non era statisticamente adeguato per valutare i costi, che, non sorprendentemente, risultavano essere senza differenze significative nei trattamenti in studio nel follow up sia a 4 sia a 12 mesi. Perciò occorre molta competenza e rigore nella interpretazione di questi risultati [41]. D'altronde, non è una soluzione giustificabile, né dal punto di vista scientifico né dal punto di vista etico, puntare sulle valutazioni economiche piggyback, più economiche e semplici, ma statisticamente meno potenti, né accontentarsi della supposizione (errata) che "assenza di evidenza è evidenza di assenza” [42].

Dal punto di vista etico, inoltre, avendo raggiunto un risultato clinico significativo di superiorità di un trattamento rispetto ad un altro, non vi sarebbero elementi per giustificare la randomizzazione e la continuazione del trial (impedendo a tutti i pazienti di accedere alla migliore terapia), fino al raggiungimento della significatività statistica anche per l'analisi economica. Tali esigenze supplementari necessarie per la valutazione farmacoeconomica verrebbero a porsi in contrasto con codici etici e lineeguida per la sperimentazione e per i comitati di etica, attualmente in vigore, e con il consenso informato dei pazienti partecipanti agli studi [43].

Per migliorare gli standard nelle analisi economiche sono state selezionate anche delle task force che hanno emanato linee guida già qualche anno fa [44] e più recentemente anche un codice etico [45]: una questione fondamentale al centro di tali documenti è l'indipendenza del ricercatore e la rivelazione del conflitto di interessi. R.G. Evans, riferendosi alle precedenti linee guida, aveva disprezzato i risultati di tali sforzi arrivando a definire la farmacoeconomia come una "pseudodisciplina impegnata a fare giochi di prestigio dentro l'esistenza della magia dei soldi" e denunciando l'inconsistenza di tali misure verso il problema strutturale degli studi: i forti interessi economici che lo sponsor e il ricercatore hanno nel cercare di ottenere gli stessi risultati [46]. 
Anche le riviste scientifiche dovrebbero essere più incisive nell' esigere la massima qualità possibile negli studi di farmacoeconomia. È stato, perciò, proposto di utilizzare standard analoghi a quelli adottati per la revisione di un articolo [47], per evitare soprattutto la citazione selettiva.

Dovrebbe fondamentalmente essere richiesto che conclusioni, modelli e possibili bias siano ben descritti, trasparenti e completamente supportati da evidenze scientifiche, condivisibili facilmente da chi è chiamato a valutare la qualità dello studio [48].

Inoltre, gli Editor dovrebbero richiedere che gli Autori rivelino tutti i possibili legami commerciali (impieghi, consulenze, viaggi pagati, proprietà di azioni, e altro ...). Le sponsorizzazioni economiche dovrebbero essere dirette all'istituzione di appartenenza e non dovrebbero dipendere dal fatto che il risultato sia favorevole al prodotto dello sponsor.

\section{CONCLUSIONI}

Come per altri ambiti della medicina, anche nella farmacoeconomia la dimensione etica, oltre che guardare ai tre diversi livelli, applicativo (dai risultati degli studi alle decisioni allocative), progettuale ("per ottenere quale risparmio per chi? Per aumentare il profitto di chi? Quale rischio prevedibile per chi?’) e intrinseco (veridicità dei dati, trasparenza e rigore scientifico dei procedimenti e delle valutazioni), dovrebbe caratterizzare in modo integrativo tutti questi aspetti: ciò vuol dire far sì che a ogni livello di attività vi sia consapevolezza che vita e salute non sono monetizzabili. L'obiettivo dell'etica in farmacoeconomia si può condensare nel rispet- to per la persona, che deve rimanere il fine e il confine di ogni scelta terapeutica come di ogni politica sanitaria.

Un altro elemento rilevante è che la valutazione farmacoeconomica costituisce anche una necessità, una obbligazione etica nell'ottica di un ottimale utilizzo e distribuzione di risorse limitate e di crescenti bisogni sanitari (principio di giustizia e di solidarietà).

Questi due aspetti, che sono anche due finalità eticamente rilevanti, sono reciprocamente confluenti e compatibili e sono entrambi necessari affinché le valutazioni di farmacoeconomia possano pervenire a un fondamentale e unico risultato, realmente ottenibile: offrire la miglior cura possibile al minor costo, nel pieno rispetto della persona e di tutte le persone, per un equo utilizzo delle risorse per tutti i cittadini.

È l'obiettivo più alto, il più arduo da raggiungere, ma esso stesso può essere, a nostro avviso, il filo conduttore ideale per lo sviluppo, l'affermazione accademica, una maggiore e migliore definizione dei processi di valutazione della farmacoeconomia. Gli strumenti indispensabili sono, dunque, già individuati, ma esigono maggiore definizione e sviluppo: un adeguato standard background/training e un approccio multidisciplinare per ottimizzare le procedure di valutazione e aumentare la veridicità e controllabilità dei risultati; le misure atte a contrastare e rivelare il conflitto di interessi perché vi sia trasparenza e credibilità; uno sguardo coerente alla qualità etica e all'umanizzazione dell'analisi farmacoeconomica che non può prescindere da una corretta valutazione della qualità della vita e di un reale rispetto delle persone.

\section{BIBLIOGRAFIA}

1. Drummond MF, Stoddart GL e Torrance GW. Metodi per la valutazione economica dei programmi sanitari. Franco Angeli. Milano 1993

2. Mazor KM, Campbell EG, Field T et al. Managed care education what medical students are telling us. Acad Med 2002;77:1128-33.

3. Anell A, Svarvar P. Pharmacoeconomics and clinical practice guidelines: a survey of attitudes in Swedish formulary committees. Pharmacoeconomics 2000; 17:175-85.

4. Hill SR, Mitchell AS Henry DA. Problems with the interpretation of pharmacoeconomics analyses: a review of submissions to the Australian Pharmaceutical Benefits Scheme. JAMA 2000;283:2116-21.

5. Ikegami N, Drummond M, Fukuhara S, et al. why has the use of health economic evaluation in Japan lagged behind that in other developed countries. Pharmacoeconomics 2002;20:Suppl. 2:1-7.

6. Hess G, Watrous M Strutton D et al. The role of pharmacoeconomics in response to globalization and increased competition in the pharmaceutical industry. In: Sam Salek, editor. Pharmacoeconomics and outcome assessment: a global issue. Euromed Communications Ltd, Haslemere 1999:119-42. 
7. Drummond MF. The future of pharmacoeconomics: bridging science and practice. Clin Ther 1996;18:969-78.

8. Rascati KL, Drummond MF, Annemans L and Davey PG. Education in Pharmacoeconomics. An International Multidisciplinary View. Pharmacoeconomics 2004; 22:139-147.

9. Kozma CM, Reeder CE, Schulz RM. Economic, clinical and humanistic outcomes: a planning model for pharmacoeconomics research. Clin Ther 1993;15:1120-32.

10. Jones D. Pharmacoeconomics and the future of the pharmaceutical industry: should they be encouraged to establish a pharmacoeconomic team? In: Sam Salek, editor. Pharmacoeconomics and outcome assessment: a global issue. Euromed Communications Ltd, Haslemere 1999:143-68.

11. Kong SX, Wertheimer AI. Outcomes research: collaboration among academic researchers, managed care organizations, and pharmaceutical manufacturers. Am J Manag Care 1988;4:28-34.

12. Copley-Merriman K, Vanscoy G, Angaran D, et al. Panel 4: education and skills needed to conduct interpret, and use economic evaluations in healthcare. Value Health 1999; 2:88-91.

13. ISPOR News. What do you consider an ideal training for the practitioner of pharmacoeconomic research?. ISPOR News 1999; 5(1). In: www.ispor.org/news/articles/0501/ifits.html

14. Paltiel D, Neumann PJ. Why training is the key to successful guideline implementation. Pharmacoeconomics 1997; 12:297-302.

15. Marsh W. Learning objectives task force: report on activities. Philadelphia (PA): ISPOR leadership meeting 2002.

16. The National Commission for the Protection of Human Subjects of Biomedical and Behavioral Research. Institutional review boards: report and recommendations. Washington, DC: DHEW Publication N.(OS) 79-008, 1979, p. 22.

17. The National Commission for the Protection of Human Subjects of Biomedical and Behavioral Research. The Belmont report: ethical principles and guidelines for the protection of human subjects of research. Washington, DC: DHEW Publication N.(OS) 78-0012,1978, p.6.

18. Levine RJ. Ethics and regulation of clinical research. 2end ed. Baltimore: Urban \& Schwarzenberg, 1986.

19. Schipper H, Clinch J. Assessment of treatment in cancer. In: Smith GT, ed., Measuring health: a practical approach. New York: John Wiley, 1988: 109-155.

20. Brock D, Quality of Life Measures in Health Care and Medical Ethics, in: Spilker B, editor, Quality of Life and Pharmacoeconomics in Clinical Trials, second edition, Lippincott- Raven Publishers, Philadelphia 1996: 497-510.

21. Maritain J, La persona umana e il bene comune, Morcelliana, Brescia 1948.

22. Sgreccia E, Bioetica, società, sanità e qualità della vita, in: Id., Manuale di bioetica. II Aspetti medico-sociali, Vita e Pensiero, Milano 2002:3-30.

23. Troidl H, Kusche J, Vestweber KH, et al. Quality of life: an important endpoint both in surgical practice and research. J Chronic Dis 1987; 40:523-528.

24. Canterbury v. Spence, 464 F 2d 72, CADC 1972.

25. Fletcher AE, Hunt BM, Bulpitt CJ. Evaluation of quality of life in clinical trials and cardiovascular disease. J Chronic Dis 1987; 40: 557-566.

26. Lasry JCM, Margolese RG, Poisson R, et al. Depression and body image following mastectomy and lumpectomy. J Chronic Dis 1987; 40:529-534.

27. Wood- Dauphinee S, Williams JI, Reintegration to normal living as a proxy to quality of life. J Chronic Dis 1987; 40: 491-499.

28. McNeil BJ, Weichselbaum R, Pauker SG. Speech and survival: trade-offs between quality and quantity of life in laryngeal cancer. N Engl J Med 1981; 305: 982-987.

29. Drummond MF. Resource allocation decisions in health care: a role for quality of life assessments? J Chronic Dis 1987; 40: 605-616.

30. A nasty for NICE (editorial). Lancet 1999; 354:1313.

31. Schuchman M. Drug firm threatens suit over MD's product review. Toronto Globe Mail. November 17, 1999

32. Wilkinson M. Cut-price medicine put at risk. Ssidney Morning Herald. December 1, 1999.

33. Friedberg M, Saffran B, Stinson TJ, Nelson W, Bennett CL, Evaluation of Conflict of Interest in Economic Analyses of New Drugs Used in Oncology, JAMA 1999, 282:1453-57. 
34. Eichenwald K, Kolata G, Drug trials hide conflicts for doctors, New York Times, May 16, 1999; sect 1:1.

35. Eichenwald K, Kolata G, A doctor's drug studies turn into fraud. New York Times. May 17, 1999; sect O:A1.

36. Hill Sr, Mitchell AS, Henry DA. Problems with the interpretation of pharmacoeconomic analyses: a review of submissions to the Australian Pharmaceutical Benefits Scheme. JAMA 2000, 283:2116-2121.

37. Udvarhelyi IS, Colditz GA, Rai A, Epstein AM, Cost-effectiveness and cost-benefit analyses in the medical literature, Ann Intern Med 1992, 116:238-244.

38. Hillman Al, Eisemberg JM, Pauly MV, et al. Avoiding bias in the conduct and reporting of cost-effectiveness research sponsored by pharmaceutical companies, N Engl J Med 1991, 324:1362-65.

39. O’Brien B, Economic evaluation of pharmaceuticals: Frankestein's monster or vampire of trials?, Med Care 1996, 34:(suppl. 12):DS99-DS108.

40. Bennett CL, Smith TJ, George SL, Hillner BE, Fleish man S, Niell HB, Problems funding economic analyses of phase III clinical trials: free-riding and the prisoner's dilemma. J Clin Oncol 1995, 13:2457-63.

41. Bower P, Byford S, Sibbad B, Ward E, King M, Lloyd M, et al. Randomised controlled trial of non-directive counselling, cognitive-behaviour therapy, and usual general practitioner care for patients with depression. II: Cost effectiveness, BMJ 2000, 321: 1389-92.

42. Altman DG, Bland JM. Statistic notes: Absence of evidence is not evidence of absence, BMJ 1995, 311:485.

43. Briggs A. Economic evaluation and clinical trials: size matters, BMJ 2000, 321:1362-1363.

44. Task Force on Principles for Economic Analysis of health Care Technology. Economic analysis of health care technology, Ann Intern Med 1995, 122:61-70.

45. Palumbo FB, Barnes R, Deverka P, Mcghan W, Mullany L, Wertheimer A, ISPOR Code of Ethics for Researchers Background Article-Report of the ISPOR Task Force on Code of Ethics for Researchers, Value in Health, 2004, 7: 111-17.

46. Evans RG, Manufacturing consensus, marketing truth: guidelines for economic evaluation, Ann Intern Med 1995, 123:59-60.

47. Kassirer JP, Angell M. The journal's policy on cost-effectiveness analysis. New Engl. J. Med, 1994, 331:669-670.

48. Rennie D, Luft HS, Pharmacoeconomic Analyses. Making Them Transparent, Making Them Credible, JAMA 2000, 283:2158-60 